\title{
Effects of local structure of neuronal networks on spiking activity in silico
}

\author{
Tuomo Mäki-Marttunen ${ }^{1,2 *}$, Jugoslava AćimoviAć ${ }^{\text {, Keijo Ruohonen², Marja-Leena Linne }}$ \\ From Twentieth Annual Computational Neuroscience Meeting: CNS*2011 \\ Stockholm, Sweden. 23-28 July 2011
}

The structure of the neuronal network, including synaptic connectivity, is the basis for information transfer in the network. Various graph-theoretic measures such as degree distribution, mean geodesic path length, clustering coefficient and motif distribution exist for analysing the structure of networks [1], and each of them captures only one perspective of the properties that are crucial regarding the activity in the network. In this work, we vary the local structure of neuronal networks and observe changes in their activity in silico, i.e. in simulations where the activity of single neurons and their interaction is modeled. The local structure is analysed through the occurrence of different motifs, i.e. different patterns of connectivity. The effect of motifs on network dynamics has been widely studied in different types of networks: from the stability point of view in networks with unspecified dynamics [2], in artificial neural networks [3], and from synchronization point of view in spiking neuronal networks [4]. Our work focuses on noise-driven neuronal networks, where the activity can be characterised by spike trains of neurons in the network, and particularly by the bursting behaviour of the network.

To study the local structure of networks we consider the occurrences of three separate connectivity patterns: (1) the bidirectional edges, (2) the loops of three nodes, and (3) the feed-forward motifs of triples of nodes. Networks with one of these three local connectivity patterns promoted are generated - we abbreviate these networks (L1), (L2) and (L3). In addition, different distancedependent networks are generated, including networks with ring topology (RT) and biologically plausible topology, obtained by the NETMORPH [5] simulator (NM). All networks except for NM have binomially distributed in-degree, as is the case with the random networks (RN) that are widely used in neuronal activity simulations. Small illustrations of these network structures are shown in Figure 1. Neuronal activity in these types of networks of size $\mathrm{N}=100$ is simulated using the model presented in [6]. The simulations show a difference in

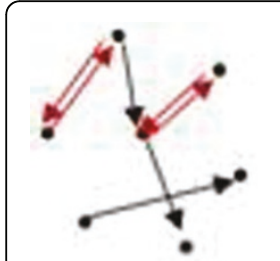

L1

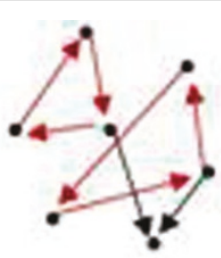

12

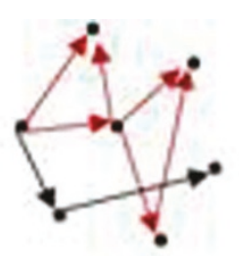

L3

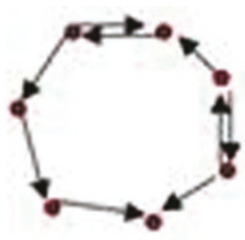

RT

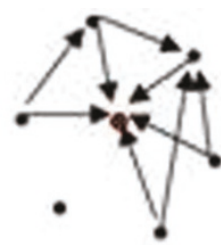

NM

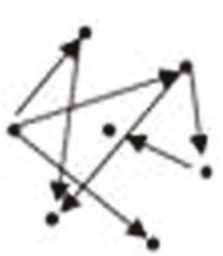

$\mathrm{RN}$

Figure 1 Different network structures. Illustration of different networks used in the present work. The red arrows and balls represent characteristics of each network, which are the following: Bidirectional edges in L1, loops of length 3 in L2, feed-forward triples in L3, nodes being placed in a ring in RT and connected to nearest neighbors, and possibility of nodes with high in-degree in NM.

\footnotetext{
* Correspondence: tuomo.maki-marttunen@tut.fi

'Department of Signal Processing, Tampere University of Technology,

Finland

Full list of author information is available at the end of the article
}

\section{() Biomed Central}

(c) 2011 Mäki-Marttunen et al; licensee BioMed Central Ltd. This is an open access article distributed under the terms of the Creative Commons Attribution License (http://creativecommons.org/licenses/by/2.0), which permits unrestricted use, distribution, and reproduction in any medium, provided the original work is properly cited. 
the activity of these networks. Preliminary results indicate, that network bursts occur more frequently in distance dependent networks RT and NM, especially in RT. Accordingly, the overall spiking frequency is high in these networks, but also in L3 networks.

\section{Author details}

'Department of Signal Processing, Tampere University of Technology, Finland. ${ }^{2}$ Department of Mathematics, Tampere University of Technology,

Finland.

Published: 18 July 2011

\section{References}

1. Boccaletti S, Latora V, Moreno Y, Chavez M, Hwang DU: Complex networks: Structure and dynamics. Phys. Rep 2006, 424(4-5):175-308.

2. Prill RJ, Iglesias PA, Levchenko A: Dynamic properties of network motifs contribute to biological network organization. PLOS Biol 2005, 3(11):1881.

3. Zhigulin VP: Dynamical motifs: building blocks of complex dynamics in sparsely connected random networks. Phys. Rev. Letters 2004, 92(23):238701.

4. Vicente R, Gollo LL, Mirasso CR, Fischer I, Pipa G: Dynamical relaying can yield zero time lag neuronal synchrony despite long conduction delays. PNAS 2008, 105(44):17157.

5. Koene RA, Tijms B, van Hees P, Postma F, de Ridder A, Ramakers GJA, van Pelt J, van Ooyen A: NETMORPH: a framework for the stochastic generation of large scale neuronal networks with realistic neuron morphologies. Neuroinformatics 2009, 7(3):195-210.

6. Tsodyks M, Uziel A, Markram H: Synchrony generation in recurrent networks with frequency-dependent synapses. J. Neurosci 2000, 20(1):50

doi:10.1186/1471-2202-12-S1-P202

Cite this article as: Mäki-Marttunen et al.: Effects of local structure of neuronal networks on spiking activity in silico. BMC Neuroscience 201112 (Suppl 1):P202.

\section{Submit your next manuscript to BioMed Central and take full advantage of:}

- Convenient online submission

- Thorough peer review

- No space constraints or color figure charges

- Immediate publication on acceptance

- Inclusion in PubMed, CAS, Scopus and Google Scholar

- Research which is freely available for redistribution

Submit your manuscript at www.biomedcentral.com/submit
Ciomed Central 